\title{
Time-Dependent Mechanics and Measures of Glial Activation and Behavioral Sensitivity in a Rodent Model of Radiculopathy
}

\author{
Sarah M. Rothman, Kristen J. Nicholson, and Beth A. Winkelstein ${ }^{1,2}$
}

\begin{abstract}
Nerve root compression induces persistent behavioral hypersensitivity and spinal glial reactivity. Viscoelastic properties of neural tissues suggest that physiologic outcomes may depend on the duration of an applied nerve root compression. This study evaluated the time-dependent properties of the root under compression in the context of pain-related behavioral and physiologic outcomes. The decrease in applied load measured by load relaxation under compression was quantified for rat cervical (C6-C8) roots in situ for durations of $30 \mathrm{sec}, 3 \mathrm{~min}$, or $15 \mathrm{~min}(\mathrm{n}=6)$. Immediately following compression, the change in the root width relative to its original width was quantified as a measure of its structural recovery. Both load relaxation and structural recovery were significantly $(p<0.05)$ correlated with duration of compression. After $30 \mathrm{sec}$ of compression, load relaxed by $22 \pm 10 \%$; increasing to $36 \pm 18 \%$ and $56 \pm 20 \%$ at 3 and $15 \mathrm{~min}$, respectively. Following $30 \mathrm{sec}, 3 \mathrm{~min}$, and $15 \mathrm{~min}$ of compression, the root recovered to $91 \pm 5 \%, 88 \pm 5$ and $72 \pm 13 \%$ of its original width, respectively. A companion in vivo study imposed these same compression durations and sham procedures to the C7 root to evaluate pain symptoms and spinal glial reactivity. Allodynia was assessed for 7 days to measure behavioral sensitivity. Immunohistochemistry and quantitative densitometry detected GFAP and OX-42 in the dorsal horn at day 7. Significant correlations were detected between compression duration and allodynia $(p<0.03)$, and astrocyte and microglial activation $(p<0.01)$. These biomechanical and glial results imply that a similar duration of compression may modulate both sustained pain and spinal glial reactivity.
\end{abstract}

Key words: allodynia; biomechanics; compression; glia; nerve root

\section{Introduction}

$\mathbf{N}$ ECK PAIN AFFECTS UP TO $71 \%$ OF INDIVIDUALS in their lifetime (Côté et al., 1998, 2000), and the annual incidence among adults is between 14 and 50\% (Côté et al., 2004; Fejer et al., 2005). Neck pain can arise from a variety of spinal tissues, including the facet joints, ligaments, and surrounding muscles, but the cervical nerve roots are at particular risk for injury due to the possibility for foraminal impingement and/or increased hydrostatic pressure, both of which can be established during rapid head and neck motions (Aldman, 1986; Bostrom et al., 1996; Eichberger et al., 2000; Nuckley et al., 2002; Örtengren et al., 1996).

Compression and direct mechanical loading to the nerve root are common sources of persistent pain (Jenis and An, 2000). Nerve root compression has been shown to produce chronic radicular symptoms that manifest clinically as pain in the shoulders, upper arms and forearms, and also weakness and numbness in the arms and hands (Abbed and Coumans,
2007; Wall and Melzack, 1994). The nerve root can be compressed by disc herniation that can result from direct spinal trauma, or more often by spinal degeneration (Atlas et al., 2005; Frymoyer, 1988; Hart et al., 1995; Ohnmeiss et al., 1997). However, neck trauma from sports and automotive injuries is sufficient to load the nerve root (Krivickas and Wilbourn, 2000; Panjabi et al., 2006; Stuber, 2005; Tominaga et al., 2006) and to induce persistent pain (Bergfield and Aulicio, 1988; Swanik et al., 1996). Lastly, slower-onset nerve root compression from foraminal stenosis can also induce pain (Cornefjord et al., 2004; Olmarker et al., 1989a). Although nerve root compression can occur from a variety of different injury scenarios, the relative local mechanical environment of the nerve root and the resulting physiologic consequences related to nociception have not been defined in relation to pain symptoms.

Soft tissues, such as nerve roots and other neural tissues that undergo loading, exhibit a time-dependent mechanical response that affects the stress-strain behavior under

\footnotetext{
${ }^{1}$ Department of Bioengineering, ${ }^{2}$ Department of Neurosurgery, University of Pennsylvania, Philadelphia, Pennsylvania.
} 
compression. Previously reported mechanical studies using several different species defined the viscoelastic properties of nervous tissue in tension and suggest that nerve roots likely experience stress relaxation, which is a decrease in stress when undergoing constant deformation over time (Chang et al., 1988; Kendall et al., 1979; Kwan et al., 1992; Miller and Chinzei, 2002). However, few studies have quantified stress relaxation in nervous tissue under compression. In a study in the pig, the applied load relaxed by over $50 \%$ when the brain was compressed (Gefen and Margulies, 2004), suggesting that the duration of an applied load may have a profound effect on the physiologic consequences of such loading to neural tissue. Data from our own laboratory have previously demonstrated a decrease in load when the nerve root is compressed to a constant deformation and held for 6 min (Hubbard et al., 2008b). However, that study did not directly quantify the relaxation in load during the nerve root compression in relation to pain-related outcomes. Despite the fact that studies have characterized the viscoelasticity of nervous tissue, no study has estimated the relaxation in load experienced by nerve roots under compression, nor has any study reported the degree to which the structure of the nerve root returns to its original geometry (structural recovery) following a transient compression. The time-dependent properties of nerve root tissue have yet to be evaluated in the context of behavioral and/or physiological outcomes related to pain.

Neuronal injury induces a host of physiologic responses that depend on the specific loading profile, such as the rate and magnitude. Animal models demonstrate that nerve root compression induces persistent behavioral hypersensitivity, decreased neuronal conduction velocity and blood flow, edema and swelling, sustained spinal glial reactivity, and other modifications of the inflammatory cascade (Garfin et al., 1990; Hubbard and Winkelstein, 2005; Igarishi et al., 2005; Olmarker et al., 1989a,b; Rothman et al., 2005). Further, many of these physiologic responses depend on the magnitude, duration, and rate of applied tissue compression (Kobayashi et al., 1993, 2005a,b; Olmarker et al., 1989a, 1989b; Pedowitz et al., 1992), implying that both magnitude and duration are important factors in modulating nerve function for compression. Although previous work has defined useful relationships between mechanics and physiologic outcomes of dysfunction, no study to date has evaluated how the duration of nerve root compression affects behavioral hypersensitivity and/or spinal glial reactivity and whether these biologic outcomes relate to specific mechanical responses at the nerve root.

The purpose of this study was to broadly characterize the overall mechanical and physiological responses following nerve root compression applied for varying durations in a rat model of radiculopathy. Accordingly, load and structural recovery of the cervical nerve root under an applied compression were used to define the mechanical response to compression for different durations. Using the same duration periods, behavioral hypersensitivity was evaluated by measuring mechanical allodynia induced after compression, and spinal glial reactivity was assessed at day 7 to define spinal responses induced by a transient compression. Applied compression was held for several different time periods in separate studies: $30 \mathrm{sec}, 3 \mathrm{~min}$, and $15 \mathrm{~min}$. Previous work with this radiculopathy model has shown that when compression is applied for $15 \mathrm{~min}$ to produce persistent allodynia the load relaxes rapidly within the first $30 \mathrm{sec}$ (Hubbard et al., 2008a). As such, this short period of applied compression was chosen as a relevant duration to quantify the effects of the early contributions of compression. The compression duration of 3 min was chosen because it is a shorter duration than when steady-state load is reached (Hubbard et al., 2008a). Finally, the 15 min of compression was also included in this study as a control since it is the duration that is known to induce persistent sustained mechanical allodynia (Hubbard et al., 2008a; Rothman and Winkelstein, 2007). In this study, the mechanical environment of nerve root compression is defined for painful loading conditions in the rat that result in persistent mechanical allodynia and spinal glial activation. The sensitivity of the mechanical and physiological outcomes to the duration of compression was evaluated by comparing the variance between each group; correlations between the duration of applied load to the cervical nerve root and each of behavioral and spinal glial responses are also tested.

\section{Methods}

Two companion studies were performed to define the relative effects of the duration of an applied compression to the cervical nerve root on both the mechanical properties of the tissue and the resulting behavioral and spinal glial responses. In both studies, cervical nerve roots were compressed at a rate of $0.005 \mathrm{~mm} / \mathrm{sec}$ to a targeted peak compressive load of $110 \mathrm{mN}$, using a previously described customized device that incorporates a stationary and a moving micro-compression platen to compress the nerve root (Hubbard et al., 2008a). For all procedures, the displacement of the translating platen during compression was measured by an LVDT ( $5 \mathrm{~mm}$ travel distance, $0.25 \%$ sensitivity; RDP, Pottstown, PA); a load cell $(0.15 \mathrm{mN}$ resolution; Omega, Stamford, CT) was coupled to the stationary platen and measured the load applied during compression. Load and displacement were continuously recorded during each compression at $10 \mathrm{~Hz}$ using LabVIEW (National Instruments, Austin, TX) and were used to quantify the mechanical response of the nerve root tissue under compression.

Rats were housed under USDA- and AAALAC-compliant conditions with a $12 / 12 \mathrm{~h}$ light-dark cycle and free access to food and water. All experimental procedures were approved by an Institutional Animal Care and Use Committee.

\section{Nerve root mechanical behavior during and after compression}

In order to define the mechanical response of the nerve root during and after an applied compression, the load response and structural geometry were measured. The amount of load relaxation under a fixed compression and the amount of structural recovery of the nerve root width immediately after release from the applied load were measured, respectively. Separate groups of rats were used for each of the different compression durations: $30 \mathrm{sec}(n=6), 3 \mathrm{~min}(n=6)$, and $15 \mathrm{~min}(n=6)$. In order to measure the relevant nerve root anatomy for the structural recovery, the nerve root had to be visualized from a lateral perspective, unobstructed by the surrounding tissue (Fig. 1). This is not possible in vivo because it requires removing the lateral structures. As such, for these studies, compression was applied to the C6-C8 cervical nerve roots immediately after the rat had expired. 


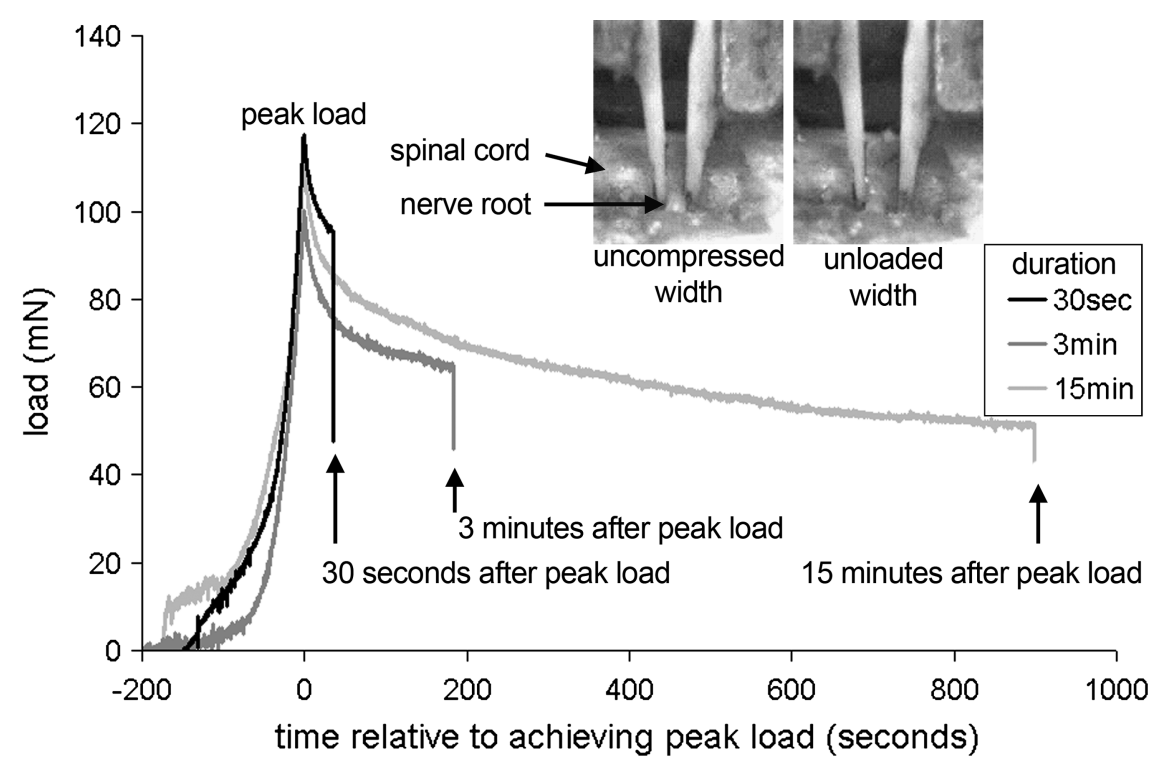

FIG. 1. Representative responses of load relaxation during a compression applied for $30 \mathrm{sec}, 3 \mathrm{~min}$, or $15 \mathrm{~min}$, with corresponding images showing the lateral view of the right dorsal nerve root exiting the spinal cord. The uncompressed and unloaded widths of the nerve root are also shown at the corresponding time points when acquired relative to the loading. The uncompressed width was calculated from the original width of the root; the final image was taken as the point at which the translating platen first moved completely off the nerve root and was used for the unloaded width.

For each compression, the nerve root was situated between the two platens of the compression device; the platens were always positioned with a gap of $1 \mathrm{~mm}$ between them, with the stationary platen flush against the root. This approach provided enough space between the platens to accommodate the nerve root and to allow the platens to safely avoid the bony structures of the surrounding vertebrae. With the platens in place, the nerve root was compressed transversely through its diameter until the prescribed load was reached and the compression was held for $30 \mathrm{sec}, 3 \mathrm{~min}$, or $15 \mathrm{~min}$. At the end of the compression period, the compression was released by returning the translating platen to its initial position. The load relaxation was estimated by measuring the load at the end of the compression period, taken just before unloading the root (Fig. 1). This final load was measured relative to the peakapplied load for each test. The degree of structural recovery in the nerve root was estimated using the width of the nerve root measured both before and after the applied compression.

The structural recovery of the nerve root width after a transient compression was used to provide a proxy to estimate the tissue's ability to return to its original shape after loading. The width of each nerve root was measured at two time points for each loading case: prior to applying the compressive load (uncompressed width) and just after the compressive load was released (unloaded width) (Fig. 1). The digital video (Qimaging, Burnaby, Canada) acquired from the lateral view was used to detect the nerve root width from images taken at the corresponding time points (Fig. 1). For each image, the width of the nerve root was measured three times and the mean was recorded. The structural recovery of the nerve root after unloading was expressed as a percent of recovery to the uncompressed width, with $100 \%$ being complete geometric recovery.

Separate one-way ANOVAs with post hoc Bonferroni correction tested for differences in the load relaxation and percent of structural recovery with $30 \mathrm{sec}, 3 \mathrm{~min}$, and $15 \mathrm{~min}$ of applied compression in order to probe for differences in the mechanical response due to the duration of applied nerve root compression. Separate linear regression models were used to test for significant correlations between: (1) the degree of load relaxation during the applied compression and (2) the amount of structural recovery following compression and the duration of compression. The significance of correlation was tested using an F-test. Significance was defined as $p<0.05$ (SYSTAT, Richmond, CA).

\section{Behavioral and spinal glial outcomes}

In vivo experiments were performed using male Holtzman rats (Harlan Sprague-Dawley, Indianapolis, IN), weighing 250-350 $\mathrm{g}$ at the start of the study, to evaluate the effects of compression duration on the resulting mechanical allodynia and spinal glial reactivity. The compressions were applied for the same duration periods as those used in the mechanical study in order to establish physiological context for the mechanical effects defined in that study. All experimental procedures were carried out under the guidelines of the Committee for Research and Ethical Issues of the International Association for the Study of Pain (Zimmermann, 1983).

All surgical procedures were performed under inhalation anesthesia (4\% isoflurane/halothane for induction, $2 \%$ for maintenance). A transient compression was applied to each rat on the right $\mathrm{C} 7$ nerve root in separate studies for $30 \mathrm{sec}$ $(n=6), 3 \mathrm{~min}(n=6), 15 \mathrm{~min}(n=4)$, or a sham exposure $(n=2)$ with no applied compression. For all compressions, the load applied was above the load threshold $(38.2 \mathrm{mN})$ previously determined to produce sustained ipsilateral mechanical allodynia if applied for $15 \mathrm{~min}$ (Hubbard et al., 2008a). In addition, previous work had shown that loads above $54.4 \mathrm{mN}$ do not produce different allodynia responses (Hubbard et al., 
2008a); for this study, applied compressive loads ranged from 73 to $146 \mathrm{mN}$. Following surgery, all wounds were closed using 3-0 polyester suture and surgical staples. Rats were allowed to recover in room air and were monitored continuously.

Following surgery, all rats were monitored for mechanical allodynia in the ipsilateral forepaw on days 1, 3, 5, and 7 to quantify the relative behavioral hypersensitivity. Prior to surgery, baseline measurements were recorded for at least two consecutive days for unoperated controls. For allodynia assessment, rats were stimulated on the plantar surface of the ipsilateral forepaw using two von Frey filaments (2 and $4 \mathrm{~g}$ ) (Stoelting Co., Wood Dale, IL). Each testing session consisted of three rounds of 10 stimulations each, separated by $10 \mathrm{~min}$. The total number of paw withdrawals was summed for each rat with each filament on each day and averaged. Overall significant differences in allodynia over time were detected using a two-factor ANOVA with repeated measures, with compression duration and postoperative time (days 1, 3, 5, 7) as the factors. A one-way ANOVA with repeated measures and post hoc Bonferroni correction was used to identify significant differences in allodynia between each duration group. Similarly, a one-way ANOVA was used to detect differences in allodynia between each group on days 1 and 7, separately. Day 1 was explicitly examined for insight into the onset of sensitivity, and day 7 for persistence of allodynia and to provide behavioral context for the glial activation data. In addition, linear regressions were used to test for correlations between the degree of mechanical allodynia at day 1, day 7 , and over the entire postoperative period (total allodynia) with respect to the duration of the applied compression for testing with both filaments. The significance $(p<0.05)$ of correlation was tested using an F-test.

Glial activation in the ipsilateral dorsal horn of the spinal cord was also assessed at day 7 after compression. Accordingly, immunohistochemical techniques detected glial fibrillary acidic protein (GFAP) as a marker of astrocytic activation and microglial reactivity was detected using OX-42 (CR3/ CD11b). Spinal cord tissue at the C7 level was harvested at day 7 after surgery for all rats that underwent behavioral monitoring in order to evaluate the effect of compression duration on spinal glial reactivity. Rats were given an overdose of sodium pentobarbital $(40 \mathrm{mg} / \mathrm{kg}$ ) and transcardially perfused with $200 \mathrm{~mL}$ of phosphate-buffered saline (PBS) followed by $300 \mathrm{~mL}$ of $4 \%$ paraformaldehyde in PBS. Spinal cord tissue was harvested and placed in $4 \%$ paraformaldehyde for $20 \mathrm{~min}$, then in $30 \%$ sucrose for 3 days at $4{ }^{\circ} \mathrm{C}$ before freeze mounting in OCT (Fisher, Fairlawn, NJ), and stored at $-80^{\circ} \mathrm{C}$.

Axial sections $(20 \mu \mathrm{m})$ were taken for free-floating immunohistochemistry. A polyclonal antibody to GFAP (Dako, Carpinteria, CA) was used as a marker of activated astrocytes. Tissue sections were blocked with normal goat serum (Vector Labs, Burlingame, CA) for 20 min followed by incubation overnight in a primary antibody directed against GFAP (1:20,000). An Alexa-594 conjugated goat anti-rabbit secondary antibody (1:1000) was used for immunofluorescent detection (Invitrogen, Carlsbad, CA). For a second set of matched axial spinal cord sections from each rat, a monoclonal antibody to OX-42 (BD Pharmingen, San Diego, CA) was used as a marker of activated microglia (Hashizume et al., 2000; Winkelstein and DeLeo, 2002; Zhuang et al., 2005). For OX-42, spinal cord slices were blocked with normal goat serum (Vector) for $20 \mathrm{~min}$ and incubated overnight in an antibody directed against OX-42 (1:500). An Alexa-546 conjugated goat anti-mouse secondary antibody (1:500) was used for immunofluorescent detection (Invitrogen). Optimal antibody dilutions for all immohistochemistry were previously determined (Rothman and Winkelstein, 2007). A negative control with no primary antibody staining was always included for verification of specificity of immunohistochemical techniques. Normal naïve tissue was also processed in each run in order to provide a baseline comparison.

Glial activation was analyzed using quantitative densitometry to detect the extent of staining and to enable quantitative and statistical comparisons. For each marker of glial reactivity, representative spinal cord sections from each rat were photographed at 100x magnification using a digital camera and stereomicroscope system equipped with Axiovision software (Zeiss, Thornwood, NY). The dorsal horn on the side ipsilateral to the injury site was assessed. Images were cropped to regions of interest $(800 \times 200$ pixels) that included the superficial lamina I-III of the dorsal horn only. Fluorescent images were inverted to analyze images for percentage of pixels above a defined threshold, with threshold pixel intensity chosen based on staining in normal tissue (Abbadie et al., 1996; Romero-Sandoval et al., 2008; Rothman and Winkelstein, 2007). Threshold pixel intensity was chosen based on staining in normal tissue. This approach gives a quantitative measure of the degree and amount of staining above what is present in normal naive tissue. Normalized tissue intensity results were averaged for each compression duration group (30 sec, $3 \mathrm{~min}, 15 \mathrm{~min}$, sham). A one-way ANOVA with post hoc Bonferroni correction was used to compare each duration group for each protein (GFAP, OX-42) separately. As with the other outcomes in this study, significant correlations between the degree of astrocyte and microglial activation and the duration of the applied compression were tested using linear regression. In addition, a linear regression model was used to test for significant correlations between mechanical allodynia measured at day 7 using both the $2 \mathrm{~g}$ and $4 \mathrm{~g}$ filaments, separately, and the degree of astrocyte and microglial activation in order to investigate relationships between the two physiologic outcomes.

\section{Results}

The two companion studies were performed to evaluate the mechanical responses of the nerve root for compressive loading and the physiological outcomes following transient nerve root compression in a coordinated approach. No significant differences were detected in the average widths of the C6, C7, and C8 nerve roots used in the in situ mechanical study (data not shown). Moreover, the uncompressed widths of the nerve roots in the mechanical and in vivo study were also not different; neither were the peak loads applied in the two studies reported here.

\section{Load relaxation during compression and structural recovery after compression}

No significant differences in the peak load applied or the linear deformation of the nerve root at peak load were measured with $30 \mathrm{sec}, 3 \mathrm{~min}$, and $15 \mathrm{~min}$ of applied compression. For the mechanical study, the mean peak load applied in each 
of the groups was $108.86 \pm 14.33 \mathrm{mN}$ for $30 \mathrm{sec}$ of compression, $110.11 \pm 19.76 \mathrm{mN}$ for $3 \mathrm{~min}$, and $118.71 \pm 15.79 \mathrm{mN}$ for $15 \mathrm{~min}$.

Regardless of the length of the duration of applied compression, the load relaxed over the period when the nerve root underwent compression (Figs. 1 and 2). After $30 \mathrm{sec}$ of applied nerve root compression, the load relaxed by $21.59 \pm 9.91 \%$ of its peak applied load. After 3 min of applied compression, the load relaxed by $35.81 \pm 18.20 \%$ of its peak load, and this increased even further to $55.99 \pm 20.20 \%$ of its peak load in the group that underwent 15 min of applied compression (Fig. 2). The amount of load relaxation observed after $3 \mathrm{~min}$ of applied load was significantly ( $p=0.038$ ) greater than that after $30 \mathrm{sec}$, and the amount of load relaxation after $15 \mathrm{~min}$ of applied load was significantly $(p<0.025)$ greater than that of both $30 \mathrm{sec}$ and $3 \mathrm{~min}$ (Fig. 2).

After $30 \mathrm{sec}$ and $3 \mathrm{~min}$ of compression, the nerve root demonstrated greater recovery than it did following compression that was applied for $15 \mathrm{~min}$ (Fig. 3). After $30 \mathrm{sec}$ and $3 \mathrm{~min}$ of applied compression, the nerve root returned to $91.0 \pm 4.9 \%$ and $87.6 \pm 5.4 \%$ of the uncompressed width, respectively (Fig. 3). No significant difference in structural recovery was observed when the compression was applied for $30 \mathrm{sec}$ or $3 \mathrm{~min}$ (Fig. 3). However, after $15 \mathrm{~min}$ of compression, the nerve root returned to only $72.4 \pm 12.6 \%$ of the corresponding uncompressed width and this was significantly $(p<0.02)$ less than the structural recovery observed after either of the $30 \mathrm{sec}$ or 3 min compression durations (Fig. 3).

Both load relaxation and structural recovery were significantly correlated with the duration of the applied compression. The degree to which the load relaxed relative to the peak load exhibited a significant $\left(p=0.049 ; \mathrm{r}^{2}=0.47\right)$ positive correlation with respect to the length of time the root was compressed. Similarly, the amount of structural recovery observed in the nerve root following compression exhibited a significant $\left(p=0.02 ; \mathrm{r}^{2}=0.53\right)$ negative correlation with the duration of applied compression.

\section{Mechanical allodynia}

Mechanical allodynia produced after $30 \mathrm{sec}, 3 \mathrm{~min}$, and $15 \mathrm{~min}$ of applied compression and sham varied significantly over the postoperative period with both the $2 \mathrm{~g}$ and $4 \mathrm{~g}$ fila- ments $(p<0.001)$ (Fig. 4). Sham procedures did not produce increases in the number of paw withdrawals compared to baseline for the different time points and testing with either filament strength.

For all of the days in which mechanical allodynia was measured, $30 \mathrm{sec}$ and $3 \mathrm{~min}$ of applied compression elicited significantly fewer paw withdrawals than did $15 \mathrm{~min}$ of applied compression (Fig. 4). After $30 \mathrm{sec}$ of compression, mechanical allodynia was significantly less than that after $15 \mathrm{~min}$ of compression for testing with both the $2 \mathrm{~g}$ filament ( $p=$ 0.042 ) and $4 \mathrm{~g}$ filament ( $p=0.03)$. Likewise, $3 \mathrm{~min}$ of compression elicited significantly less mechanical allodynia than that with $15 \mathrm{~min}$ of compression, for both the $2 \mathrm{~g}$ filament $(p=0.03)$ and $4 \mathrm{~g}$ filament $(p=0.024)$. Immediately (day 1$)$ after $15 \mathrm{~min}$ of nerve root compression, allodynia was significantly $(p<0.005)$ greater than that induced by $30 \mathrm{sec}$ of compression for testing with both filaments (Fig. 4). Allodynia after 15 min of nerve root compression was also significantly $(p=0.026)$ greater than allodynia produced after $3 \mathrm{~min}$ of compression at this time point for testing with the $2 \mathrm{~g}$ filament. However, by day 7 after $15 \mathrm{~min}$ of nerve root compression, allodynia was significantly $(p<0.008)$ greater than only that response elicited after compression applied for $30 \mathrm{sec}$; this was true for testing with both filaments (Fig. 4).

Mechanical allodynia after $30 \mathrm{sec}$ or $3 \mathrm{~min}$ of applied compression was not significantly different from sham for any time point probed or for either filament. In addition, there were no significant differences in mechanical allodynia in the ipsilateral paw detected between $30 \mathrm{sec}$ and $3 \mathrm{~min}$ of applied compression at any time point for either filament (Fig. 4). Contralateral mechanical allodynia exhibited a similar trend in that allodynia elicited by the 15 min of compression was elevated over allodynia measured in the shorter duration groups; however the results were not robust (data not shown).

The degree of mechanical allodynia elicited by compression was significantly correlated with the duration of applied compression for testing with both the $2 \mathrm{~g}$ and $4 \mathrm{~g}$ filaments. Mechanical allodynia measured on days 1 and 7 , as well as the total allodynia using a $2 \mathrm{~g}$ von Frey filament, exhibited a positive linear correlation $\left(p<0.03 ; r^{2}>0.51\right.$; Fig. 5). Similarly, mechanical allodynia measured at day 7 using a $4 \mathrm{~g}$ von Frey filament also exhibited a significant positive linear

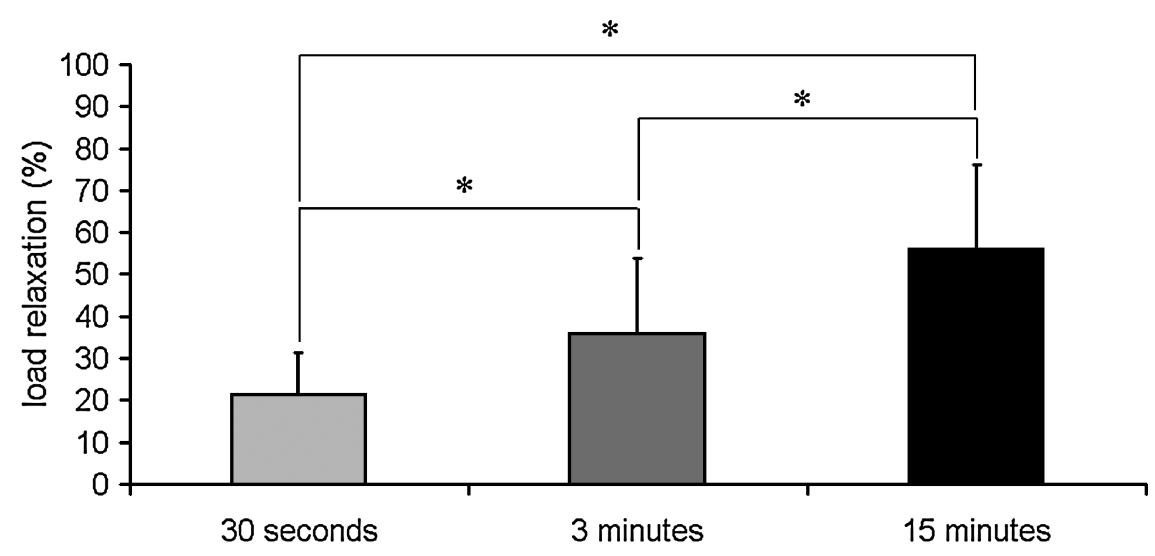

FIG. 2. Quantification of the mean percent of load relaxation experienced by the nerve root measured at $30 \mathrm{sec}$, $3 \mathrm{~min}$, or $15 \mathrm{~min}$ after the peak load. Percent relaxation was measured relative to the peak load for each case and is expressed here as mean \pm standard deviation. *Significant difference between groups. 


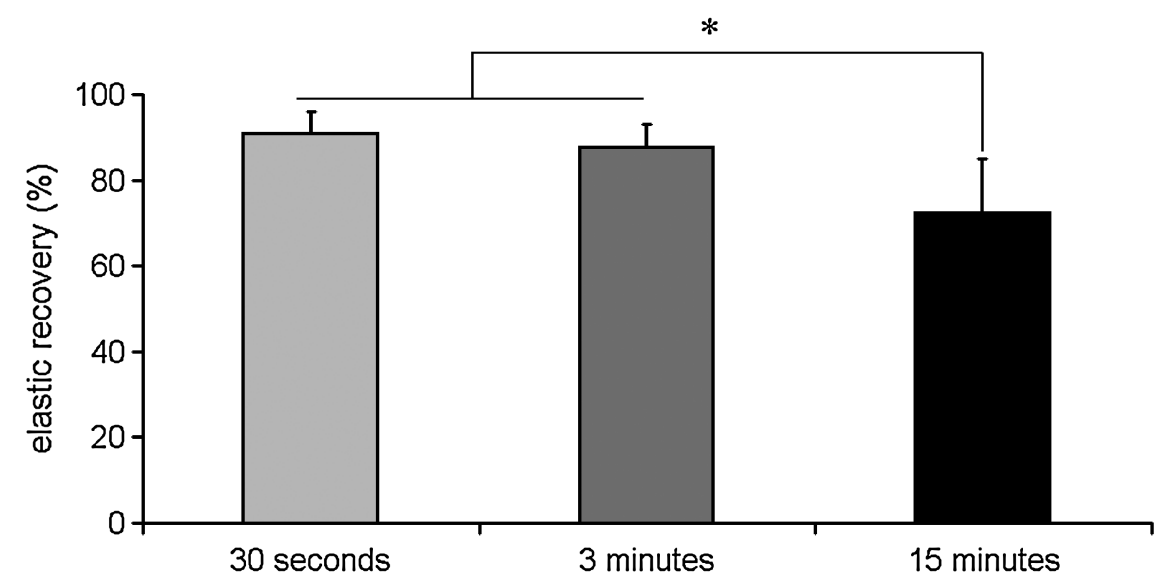

FIG. 3. Structural recovery of the nerve root after $30 \mathrm{sec}, 3 \mathrm{~min}$, or $15 \mathrm{~min}$ of applied compression. Recovery is expressed as a percent return to original width (mean \pm standard deviation); $100 \%$ represents full recovery of the nerve root after compression to its uncompressed width. *Significant difference.
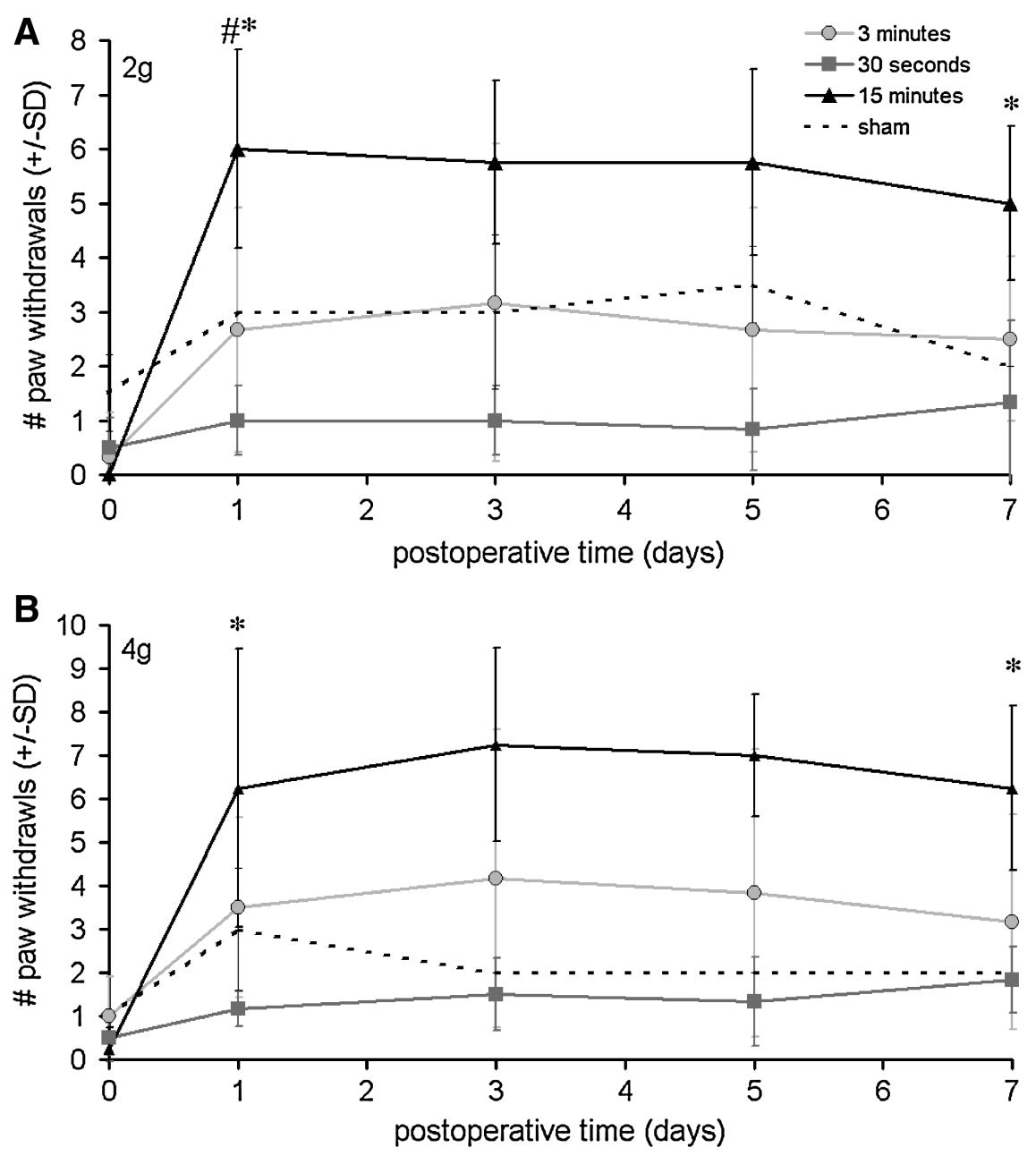

FIG. 4. Average mechanical allodynia assessed in the ipsilateral forepaw after $30 \mathrm{sec}, 3 \mathrm{~min}$, or $15 \mathrm{~min}$ of applied nerve root compression, as well as sham. Allodynia is quantified as the number of paw withdrawals for stimulation with a $2 \mathrm{~g}$ (A) and $4 \mathrm{~g}$ von Frey filament (B). All groups were significantly different from each other over time for both filaments. There was no significant difference between $30 \mathrm{sec}$ and $3 \mathrm{~min}$ of compression. "Significant difference between $15 \mathrm{~min}$ and $3 \mathrm{~min}$ of compression; *significant differences between $15 \mathrm{~min}$ and $30 \mathrm{sec}$ of compression at time points indicated; SD, standard deviation. The greater the number of paw withdrawals, the greater the behavioral sensitivity. 


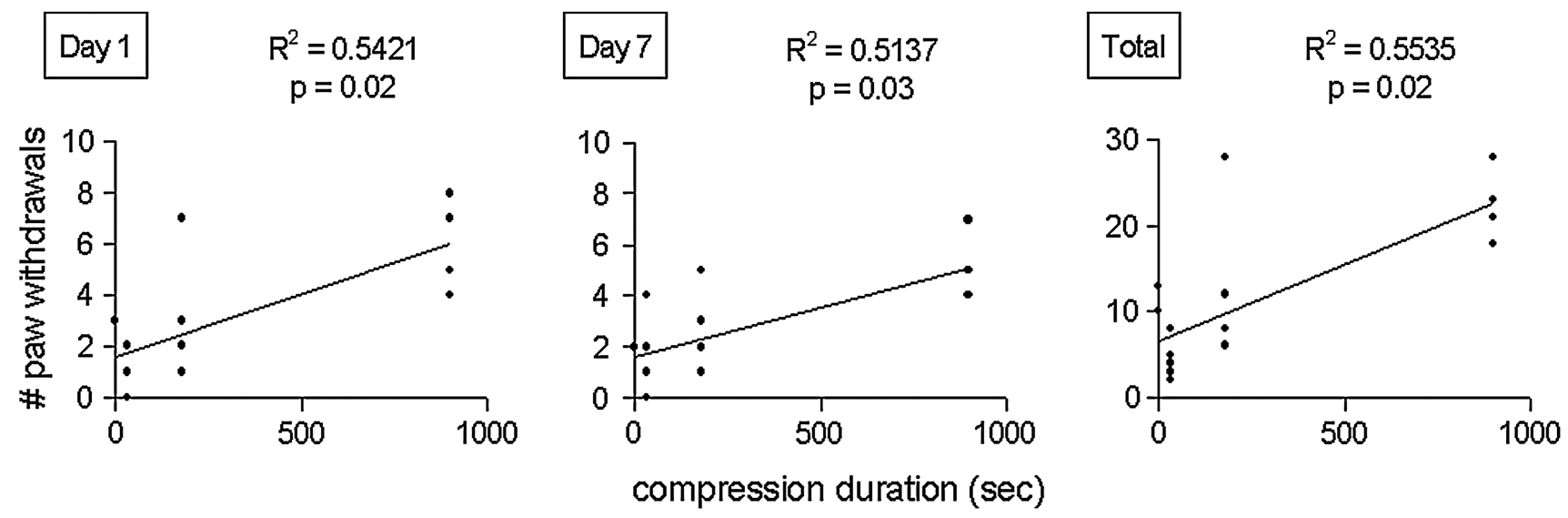

FIG. 5. Linear regression correlation between duration of compression and number of paw withdrawals for stimulation with a $2 \mathrm{~g}$ von Frey filament at days 1 and 7 , as well as total allodynia.

correlation $\left(p=0.03 ; \mathrm{r}^{2}=0.52\right)$. While not significant, mechanical allodynia measured using a $4 \mathrm{~g}$ von Frey at day 1 and total allodynia both exhibited strong positive linear correlations $\left(r^{2}=0.45\right.$ and $r^{2}=0.44$, respectively).

\section{Spinal glial reactivity}

Spinal GFAP expression in the ipsilateral dorsal horn showed a graded response relative to the duration of the applied compression, with GFAP expression increasing with longer applied compression hold times (Figs. 6 and 7). After compression applied for $30 \mathrm{sec}$, spinal GFAP expression in the ipsilateral dorsal horn was not significantly different from normal or sham. However, after $3 \mathrm{~min}$ of applied root compression, GFAP was significantly $(p=0.02)$ increased over normal (Fig. 7). When compression was applied for $15 \mathrm{~min}$, it induced even further and more robust GFAP expression in the dorsal horn at day 7 (Fig. 7). After $15 \mathrm{~min}$ of applied nerve root compression, GFAP expression was significantly $(p<0.001)$ elevated over the GFAP expression induced by $30 \mathrm{sec}$ and $3 \mathrm{~min}$ compressions, as well as over sham and normal $(p<0.001)$.

In contrast, spinal OX-42 expression did not demonstrate the same graded response according to the compression duration as was observed with GFAP (Figs. 6 and 7). OX-42 expression after nerve root compressions applied for either $30 \mathrm{sec}$ or $3 \mathrm{~min}$ was not significantly increased in the ipsilateral spinal cord above that of normal or sham (Fig. 7). Yet, ipsilateral OX-42 expression on day 7 after 15 min of nerve root compression was significantly $(p<0.001)$ increased over spinal expression of OX-42 elicited by both $30 \mathrm{sec}$ and $3 \mathrm{~min}$ compression, as well as sham and normal ( $p<0.001)$ (Fig. 7).

When examining the spinal glial reactivity in terms of the compression duration, significant correlations were found between the duration of the applied compression and both spinal glial outcomes. Both GFAP and OX-42 expression in the ipsilateral dorsal horn on day 7 were significantly $(p<0.001$; $\mathrm{r}^{2}=0.65$ and $\mathrm{r}^{2}=0.78$, respectively) and positively correlated with the applied compression duration. GFAP and OX42 expression in the contralateral dorsal horn was not significantly elevated over normal for either sham or the three duration groups (data not shown). Despite the fact that both mechanical allodynia and glial activation were each significantly correlated with the duration of the applied compression (Fig. 5), no significant correlations were detected between either astrocyte activation or microglial activation and mechanical allodynia for testing with either the $2 \mathrm{~g}$ or $4 \mathrm{~g}$ filament at day $7\left(\mathrm{r}^{2}<0.25\right)$.

\section{Discussion}

Currently, there are no studies that quantify the load experienced by neural tissues in vivo for compression during, or related to, painful loading. To our knowledge, this is the first study to measure both the load relaxation response of a nerve root while held under compression and its structural recovery immediately after the release of loading. The applied loads in this study relaxed continuously over the period that the nerve root was compressed (Fig. 1), such that the amount of load relaxation significantly increased with longer durations of compression (Fig. 2). However, this same graded response due to compression duration was not observed with structural recovery; the degree of recovery was not changed following $30 \mathrm{sec}$ or $3 \mathrm{~min}$ of applied compression, but was significantly reduced following $15 \mathrm{~min}$ (Fig. 3). Similarly, $15 \mathrm{~min}$ of compression induced sustained mechanical allodynia that was significantly greater than that seen in these same two groups, which remained unchanged from sham (Fig. 4). Interestingly, spinal microglial reactivity followed the same grading as mechanical allodynia, with no difference from controls for the shorter durations; while expression of the astrocytic marker, GFAP, on the other hand, was graded, in a manner similar to that of load relaxation (Figs. 2 and 7). These studies suggest that behavioral hypersensitivity may be related to the structural integrity of the nerve root following compression and that the duration of injury modulates spinal glial activation.

The nerve root mechanical response exhibited was timedependent for a constant deformation (Figs. 1 and 2). After $15 \mathrm{~min}$ of compression, the load relaxed by $56 \%$ percent of its peak load (Fig. 2), which is similar to previous studies of neural tissue in compression (Gefen and Margulies, 2004) and tension (Driscoll et al., 2002, Wall et al., 1991), and only recovered to approximately $70 \%$ of its original width (Fig. 3). Although this study did not specifically investigate the mechanism for these time-dependent properties, it is possible that 

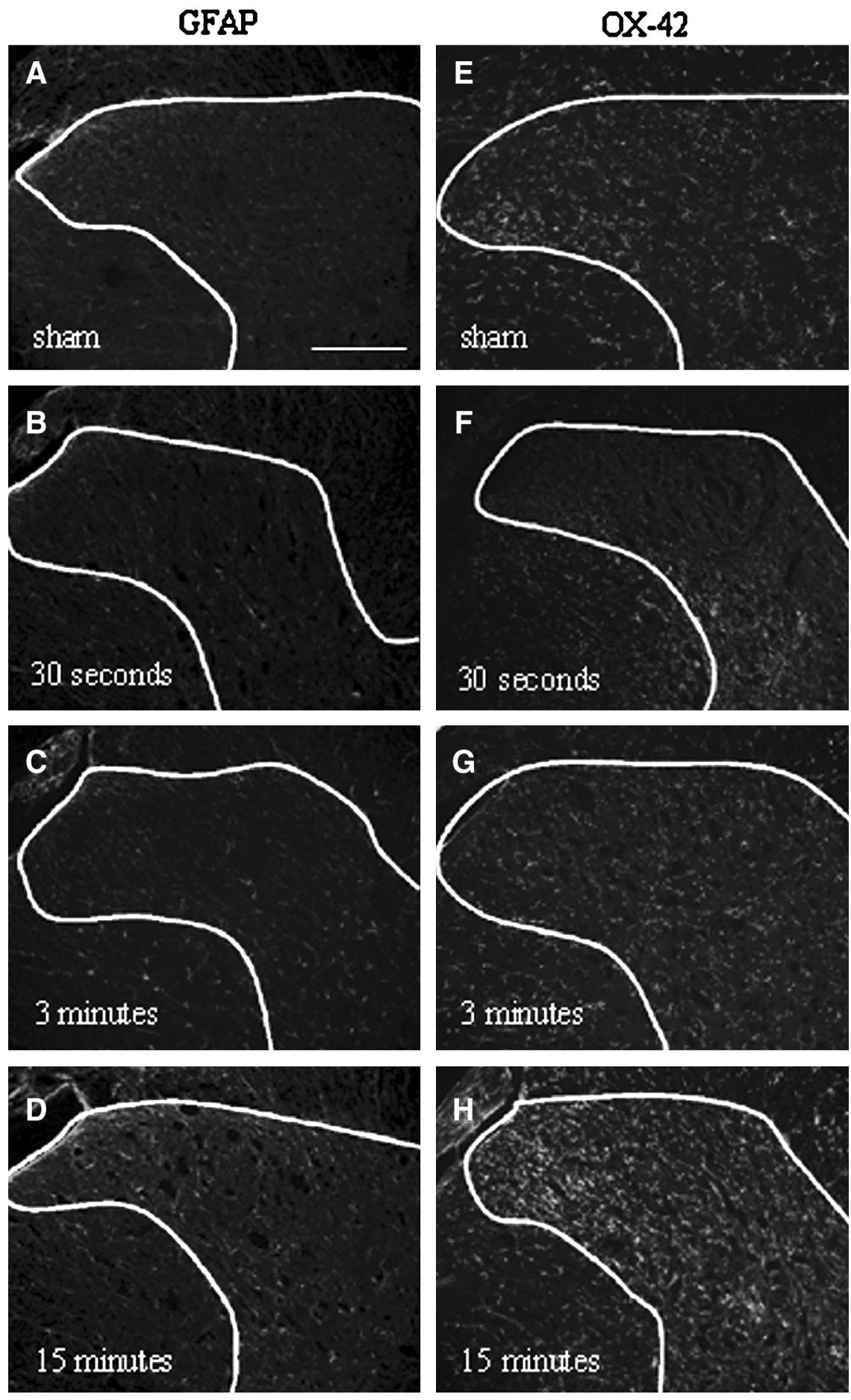

FIG. 6. Representative ipsilateral C7 spinal cord sections showing superficial laminae stained against GFAP (A-D) and OX$42(\mathrm{E}-\mathrm{H})$ at day 7 after sham, $30 \mathrm{sec}, 3 \mathrm{~min}$, or $15 \mathrm{~min}$ of compression. GFAP expression increases with longer durations of compression; OX-42 expression only increases after $15 \mathrm{~min}$ of applied compression. The grey and white matter are outlined. Scale bar, $100 \mu \mathrm{m}$.

the observed load relaxation and loss of structural recovery following 15 min of compression may be due to a breakdown in the structural integrity of the axons. All loads applied in this study were above the load threshold $(31.6 \mathrm{mN})$ previously determined to significantly reduce neurofilament expression following nerve root compression (Hubbard and Winkelstein, 2008), which is also similar to the load required to induce persistent behavioral hypersensitivity. However, although that study implied that a breakdown in axonal transport may be related to, or associated with, the onset of behavioral hypersensitivity, the compression was held for $15 \mathrm{~min}$ and shorter hold times were not examined. In this study, behavioral sensitivity, structural recovery, and glial activation were all significantly changed following a 15 min compression, but 

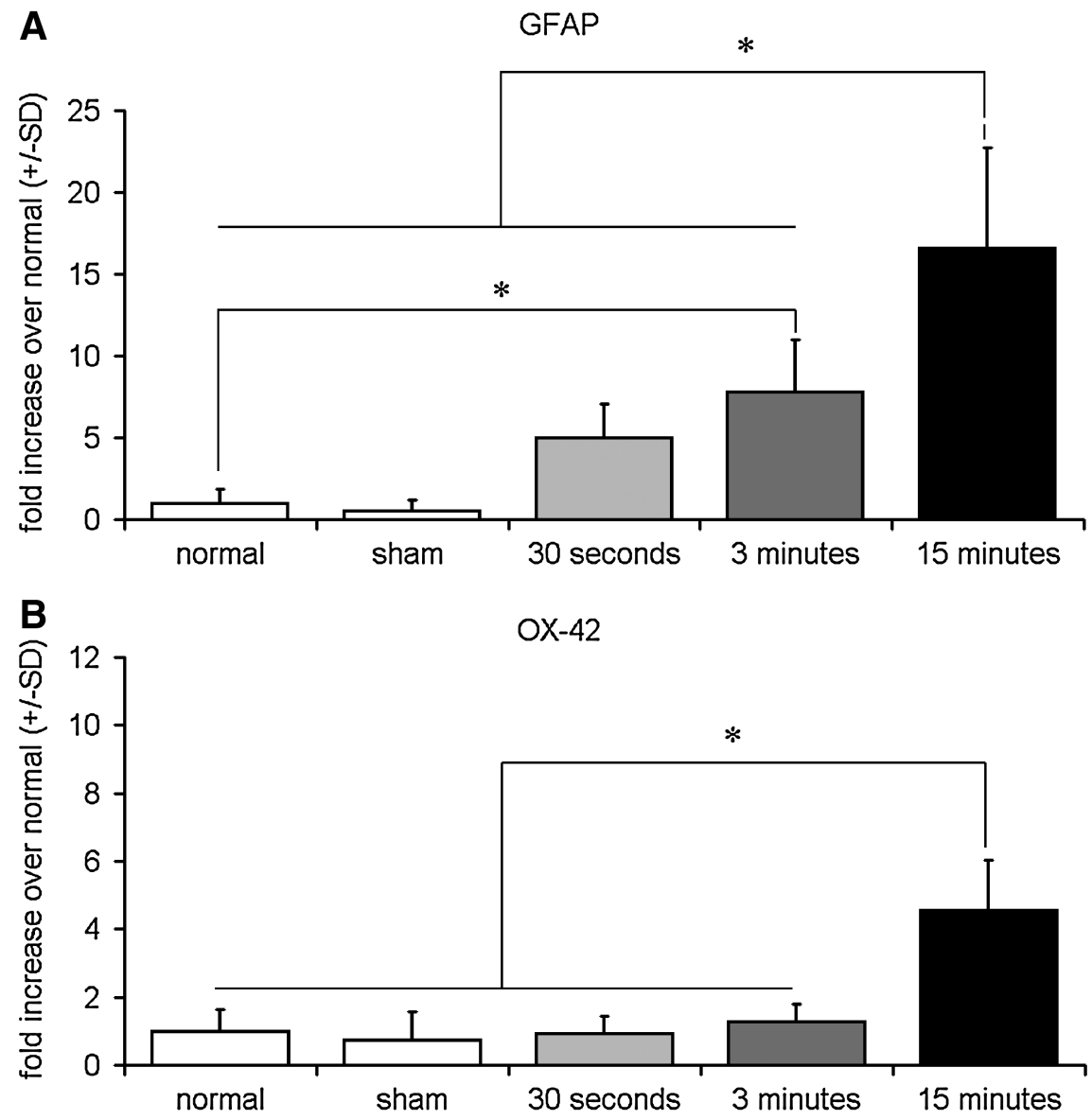

FIG. 7. Automated densitometry quantifying the average percentage mean \pm standard deviation (SD) of positive pixels reactive for GFAP (A) and OX-42 (B) staining at day 7 following compression. Normal and sham expression levels are shown relative to expression after $30 \mathrm{sec}, 3 \mathrm{~min}$, or $15 \mathrm{~min}$ of compression. ${ }^{*}$ Significant differences between groups.

were unchanged for $30 \mathrm{sec}$ and $3 \mathrm{~min}$ of compression (Figs. $3-7)$, suggesting that pain-related outcomes and structural integrity of the nerve root may be related to each other and supports the hypothesis that structural damage resulting in the loss of axonal transport may, in fact, be associated with persistent behavioral sensitivity.

Evaluation of other behavioral measures may provide a more discriminating assessment of changes in the behavioral sensitivity following $30 \mathrm{sec}$ and $3 \mathrm{~min}$ of compression that were not significantly different from each other in this study. Nonetheless, previous work has shown that the method of quantifying allodynia used in this study can distinguish behavioral differences for this particular injury in a way that is similar to using a threshold method (Hubbard and Winkelstein, 2005). Interestingly, allodynia elicited after $30 \mathrm{sec}$ of compression was slightly, but not significantly, lower than the response for the sham group; yet, when compared to previously reported studies, allodynia measured in the $30 \mathrm{sec}$ group does lie within the range reported for sham groups for this same cervical radiculopathy model; so this marginal difference can be attributed to the inherent variability in in vivo studies (Hubbard et al., 2008a; Hubbard and Winkelstein, 2005; Rothman and Winkelstein, 2007). Future studies investigating microscopic changes of the nerve root and additional measures of behavioral changes with respect to the duration of compression would provide further insight into the rela- tionship between the structural responses measured macroscopically here, and the associated cellular and behavioral responses in the context of the mechanical environment of the applied compression.

Local structural damage to the nerve root may be irrelevant compared to the physiological damage incurred by the tissue due to any ischemic insults imposed when the root is compressed and blood flow is eliminated. Previous work has shown that blood flow in the nerve is continuously reduced during an applied compression to a canine lumbar nerve root held for one hour (Yoshizawa et al., 1989), suggesting that there may an appreciable loss of blood flow for the longer compressions induced in this study. Furthermore, blood flow in the affected nerve root in patients with painful disc herniation was observed to increase in patients whose pain resolved after discectomy compared to patients who did not experience a relief of symptoms (Hida et al., 2003), indicating that behavioral hypersensitivity following a nerve root compression may be due, at least in part, to blood flow restriction in the nerve root. Although the current study did not quantify stress, $200 \mathrm{mmHg}(0.026 \mathrm{MPa})$ is known to completely block blood flow in the nerve root of the pig (Olmarker et al., 1989b). In fact, just $2 \mathrm{sec}$ of applied transient compression to the porcine nerve root can reduce blood flow by as much as $26 \%$ over a $3 \mathrm{~h}$ period, implying that although compression is transient, the reduction in blood flow can be sustained (Igarishi 
et al., 2005). Reductions in nerve conduction and initiation of local inflammation may be incurred by ischemia rather than by mechanical damage from the compression itself. The presence of behavioral hypersensitivity after $15 \mathrm{~min}$ of applied compression but not after $30 \mathrm{sec}$ or $3 \mathrm{~min}$ in the current study (Fig. 4) implies that the nerve root may be able to tolerate at least $3 \mathrm{~min}$ of interrupted blood flow without sustaining physiologic damage.

Nerve root compression applied for shorter periods of times did not induce spinal glial reactivity different from sham responses, despite sustained modifications in both GFAP and OX-42 reactivity following a painful $15 \mathrm{~min}$ compression (Figs. 6 and 7). Although it is not entirely clear how the mechanical signals of nerve root compression are transduced to induce glial reactivity in the spinal cord after nerve root compression, it has been shown that, after transient nerve root compression, neurons in the dorsal horn display increased action potential durations and altered release of excitatory amino acid neurotransmitters, and both astrocytes and microglia react to these types of changes (Cui et al., 1997; DeLeo et al., 2006; Hogan, 2007; Kreutzberg, 1996; Newman, 2003; Sapunar et al., 2005). Previous studies of nerve root compression in both the cervical and lumbar regions of the spine show that spinal expression of GFAP does not depend on the magnitude of compression (Hubbard and Winkelstein, 2005; Winkelstein and DeLeo, 2002). In contrast, those same studies indicate that spinal microglial activation, as measured by $\mathrm{OX}$ 42 , is modulated by the severity of the injury, as quantified by the amount of tissue deformation and compression load (Hubbard and Winkelstein, 2005; Winkelstein and DeLeo, 2002). In the current study, GFAP expression following $3 \mathrm{~min}$ of compression was significantly elevated over normal; however, there was no significant difference found in the expressions of GFAP after the shorter compressions (Fig. 6). Taken together, these studies suggest that longer-term spinal astrocytic reactivity may be more sensitive to the duration of the insult (or potential ischemic contributions) than to the actual tissue loading severity, and that microglial activation may be driven by mechanical deformation to the nerve root, with both the injury severity and duration providing integrated contributions. Regardless, additional studies explicitly investigating the interaction between the severity and duration of a mechanical insult are needed to determine the significance of these factors in pain-related physiologic outcomes.

In this study, the mechanical properties of the nerve root measured in situ were used to estimate the mechanical response of the nerve root in vivo (Figs. 2 and 3). While nerve root tissue may behave differently in situ and in vivo, these differences appear to be marginal for the specific loading conditions used in this study. A quasilinear viscoelastic mathematical model of nerve root tissue developed using compression studies in anesthetized rats (Hubbard et al., 2008b) predicted the degree of load relaxation following $30 \mathrm{sec}, 3 \mathrm{~min}$, and 15 min comparable to the load relaxation measured in this in situ study. While that mathematical model was originally developed from studies of nerve root compression for a duration of $6 \mathrm{~min}$, the viscoelastic parameters derived from that work do predict the load relaxation following $15 \mathrm{~min}$ of compression within the $95 \%$ confidence interval of load relaxation measured in vivo in nerve root compression studies (Hubbard et al., 2008a), thus validating the model for longer hold times. Also, the mechanical properties of other neural tissues have been previously shown to not vary between the immediately expired in situ and in vivo conditions (Gefen and Margulies, 2004); therefore, the estimates of load relaxation and structural recovery here are taken to provide acceptable estimates of the mechanical responses for similar compressions applied in vivo. However, further studies defining the mechanics of neural tissues in vivo are needed in order to clarify the relationship between neural trauma and physiologic responses in the context of pain.

Glial reactivity was probed at day 7 only (Figs. 5 and 6), and does not provide any insight into the early spinal glial responses for $30 \mathrm{sec}$ and $3 \mathrm{~min}$ of applied compression. However, previous work with this model has shown that GFAP expression is not changed between days 1 and 7 following a nerve root compression and that OX-42 is not significantly elevated over sham at day 1 for a $15 \mathrm{~min}$ compression (Rothman and Winkelstein, 2007). Therefore, it is unlikely that such responses will be elevated at day 1 following a $30 \mathrm{sec}$ or 3 min compression. However, spinal responses at early time points may provide insight into how such cellular responses depend on the mechanical environment at injury and also explain the behavioral outcomes in the context of spinal inflammation. Lastly, the current studies begin to quantify the load relaxation of the nerve root, but do not quantify changes in the nerve root geometry in all three dimensions due to difficulties in clearly visualizing the nerve root from other anatomic perspectives; the surgical exposure and placement of the compression platens partially obscure the dorsal view, but do currently provide the most controlled and repeatable method for applying compression in the rat cervical spine. Therefore, it was not possible to estimate the associated stress or strain of the tissue; it remains to be seen if the stress that induces persistent hypersensitivity is larger than that required to induce physiological and/or functional changes in the nerve root. Nonetheless, this work provides the foundation for understanding the role of duration of nerve root compression on the mechanical properties of the tissue, as well as the resulting pain-related behavior and spinal glial outcomes.

\section{Acknowledgments}

This work was funded by grant support from the Catharine D. Sharpe Foundation and an NSF-GOALI supplement.

\section{Author Disclosure Statement}

No competing financial interests exist.

\section{References}

Abbadie, C., Brown, J., Mantyh, P., and Basbaum, A. (1996). Spinal cord substance $P$ receptor immunoreactivity increases in both inflammatory and nerve injury models of persistent pain. Neuroscience 70, 201-209.

Abbed, K., and Coumans, J. (2007). Cervical radiculopathy: pathophysiology, presentation and clinical evaluation. Neurosurgery 60, S1-29.

Aldman, B. (1986). An analytical approach to the impact biomechanics of head and neck injury. Am. Assoc. Auto. Med. $446-454$.

Atlas, S., Keller, R., Wu, Y., Deyo, R., and Singer, D. (2005). Long-term outcomes of surgical and nonsurgical management of sciatica secondary to a lumbar disc herniation: 10 year results from the Maine lumbar spine study. Spine 30, 927-935. 
Bergfield, T., and Aulicio, P. (1988). Variation of the deep motor branch of the ulnar nerve at the wrist. J. Hand Surg. Am. 13(3), 368-369.

Bostrom, O., Svensson, M., Aldman, B., Hansson, H., Haland, Y., Lovsund, P., Seeman, T., Suneson, A., Saljo, A., and Ortengren, T. (1996). A new neck injury criterion candidate-based on injury findings in the cervical spinal ganglia after experimental neck extension trauma. International IRCOBI Conference on the Biomechanics of Impact. pp. 123-136.

Chang, G.L., Hung, T.K., and Feng, W.W. (1988). An in-vivo measurement and analysis of viscoelastic properties of the spinal cord of cats. J. Biomech. Eng. 110, 115-112.

Cornefjord, M., Nyberg, F., Rosengren, L., and Brisby, H. (2004). Cerebrospinal fluid biomarkers in experimental spinal nerve root injury. Spine 29, 1862-1868.

Côté, P., Cassidy, J., and Carroll, L. (1998). The Saskatchewan health and back pain survey: the prevalence of neck pain and related disability in Saskatchewan adults. Spine 23(15), 16891698.

Côté, P., Cassidy, J., and Carroll, L. (2000). The factors associated with neck pain and its related disability in the Saskatchewan population. Spine 25(9), 1109-1117.

Côté, P., Cassidy, J., Carroll, L., and Kristman, V. (2004). The annual incidence and course of neck pain in the general population: a population-based cohort study. Pain 112(3), 267-273.

Cui, J., O'Connor, W., Ungerstedt, U., Linderoth, B., and Myerson, B. (1997). Spinal cord stimulation attenuates augmented dorsal horn release of excitatory amino acids in the mononeuropathy via a GABAergic mechanism. Pain 73, 87-95.

DeLeo, J., Tawfik, V., and LaCroix-Fralish, M. (2006). The tetrapartite synapse: path to CNS sensitization and chronic pain. Pain 122, 17-21.

Driscoll, P., Glasby, M., and Lawson, G. (2002). An in vivo study of peripheral nerves in continuity: biomechanical and physiological responses to elongation. J. Orthop. Res. 20, 370-375.

Eichberger, A., Darok, M., Steffan, H., Leinzinger, P.E., and Bostrom, O., and Svensson, M.Y. (2000). Pressure measurements in the spinal canal of post-mortem human subjects during rearend impact and correlation of results to the neck injury criterion. Accid. Anal. Prev. 32(2), 251-260.

Fejer, R., Jordan, A., and Hartvigsen, J. (2005). Categorising the severity of neck pain: establishment of cut-points for use in clinical and epidemiological research. Pain 119(1-3), 176-182.

Frymoyer, J. (1988). Back pain and sciatica. N. Engl. J. Med. 318, 291-300.

Garfin, S., Cohen, M., Massie, J., Abitol, J., Swenson, M., Myers, R., and Rydevik, B. (1990). Nerve-roots of the cauda equina. The effect of hypotension and acute graded compression on function. J. Bone Joint Surg. Am. 72, 1185-1192.

Gefen, A., and Margulies, S. (2004). Are in vivo and in situ brain tissues mechanically similar? J. Biomech. 37, 1339-1352.

Hart, L., Deyo, R., and Cherkin, D. (1995). Physician office visits for low back pain. Frequency, clinical evaluation, and treatment patterns from a U.S. national survey. Spine 20, 11-19.

Hashizume et al., 2000.

Hida, S., Naito, M., and Kubo, M. (2003). Intraoperative measurements of nerve root blood flow during discectomy for lumbar disc herniation. Spine 28, 85-90.

Hogan, Q. (2007). Role of decreased sensory neuron membrane calcium currents in the genesis of neuropathic pain. Croat. Med. J. 48, 9-21.

Hubbard, R., Chen, Z., and Winkelstein, B. (2008a). Transient cervical nerve root compression modulates pain: Load thresh- olds for allodynia and sustained changes in spinal neuropeptide expression. J. Biomech. 41:677-685.

Hubbard, R., Quinn, K., Martinez, J., and Winkelstein, B. (2008b). The role of graded nerve root compression on axonal damage, neuropeptide changes, and pain-related behaviors. STAPP Car Crash J. 52.

Hubbard, R., and Winkelstein, B. (2005). Transient cervical nerve root compression in the rat induces bilateral forepaw allodynia and spinal glial activation: mechanical factors in painful neck injuries. Spine 30, 1924-1932.

Hubbard, R., and Winkelstein, B. (2008). Dorsal root compression produces myelinated axonal degeneration near the biomechanical thresholds for mechanical behavioral hypersensitivity. Exp. Neurol. 212, 482-489.

Igarishi, T., Yabuki, S., Kikuchi, S., and Myers, R. (2005). Effect of acute nerve root compression on endoneurial fluid pressure and blood flow in rat dorsal root ganglia. J. Orthop. Res. 23, 420-424.

Jancalek, R., and Dubovy, P. (2007). An experimental animal model of spinal root compression syndrome: an analysis of morphological changes of myelinated axons during compression radiculopathy and after decompression. Exp. Brain Res. 179, 111-119.

Jenis, L., and An, H. (2000). Neck pain secondary to radiculopathy of the fourth cervical root: an analysis of 12 surgically treated patients. J. Spinal Disord. 13, 345-349.

Kendall, J., Stokes, I., O'Hara, J., and Dickson, R. (1979). Tension and creep phenomena in peripheral nerve. Acta Orthop. Scand. 50, 721-725.

Kobayashi, S., Yoshizawa, H., Hachiya, Y., Ukai, T., and Morita, T. (1993). Vasogenic edema induced by compression injury to the spinal nerve root. Distribution of intravenously injected protein tracers and gadolinium-enhanced magnetic resonance imaging. Spine 18, 1410-1424.

Kobayashi, S., Sasaki, S., Shimada, S., Kaneyasu, M., Mizukami, Y., Kitade, I., Ogawa, M., Kawahara, H., Baba, H., and Yoshizawa, H. (2005a). Changes of calcinonin gene-related peptide in primary sensory neurons and their central branch after nerve root compression of the dog. Arch. Phys. Med. Rehabil. 86, 527533.

Kobayashi, S., Baba, H., Uchida, K., Shimada, S., Negoro, K., Takeno, K., Yayama, T., Yamada, S., and Yoshizawa, H. (2005b). Localization and changes of intraneural inflammatory cytokines and inducible-nitric oxide induced by mechanical compression. J. Orthop. Res. 23, 771-778.

Kreutzberg, G. (1996). Microglia: a sensor for pathological events in the CNS. Trends Neurosci. 19, 312-318.

Krivickas, L., and Wilbourn, A. (2000). Peripheral nerve injures in athletes: a case series of over 200 injuries. Semin. Neurol. 20(2), 225-232.

Kwan, M., Wall, E., Massie, J., and Garfin, S. (1992). Strain, stress and stretch of peripheral nerve: rabbit experiments in vitro and in vivo. Acta Orthop. Scand. 63, 267-272.

Luan, F., Yang, K., Deng, B., Begeman, P., Tashman, S., and King, A. (2000). Qualitative analysis of neck kinematics during low-speed rear-end impact. Clin. Biomech. 15, 649657.

Miller, K., and Chinzei, K. (2002). Mechanical properties of brain tissue in tension. J. Biomech. 35(4), 483-490.

Newman, E. (2003). New roles for astrocytes: regulation of synaptic transmission. Trends Neurosci. 26, 536-542.

Nuckley, D., Konodi, M., Raynak, G., Ching, R., and Mirza, S. (2002). Neural space integrity of the lower cervical spine. Effect of normal range of motion. Spine 27, 587-595. 
Ohnmeiss, D., Vanharanta, H., and Ekholm, J. (1997). Degree of disc disruption and lower extremity pain. Spine 22, 1600-1605.

Olmarker, K., Rydevik, B., and Holm, S. (1989a). Edema formation in spinal nerve roots induced by experimental, graded compression. An experimental study on the pig cauda equina with special reference to differences in effects between rapid and slow onset of compression. Spine 14(6), 569-573.

Olmarker, K., Rydevik, B., Holm, S., and Bagge, U. (1989b). Effects of experimental graded compression on blood flow in spinal nerve roots. A vital microscopic study on the porcine cauda equina. J. Orthop. Res. 7, 817-823.

Ortengren, T., Hansson, H.A., Lovsun, P., Svensson, M.Y., Suneson, A., and Saljo, A. (1996). Membrane leakage in spinal ganglion nerve cells induced by experimental whiplash extension motion: a study in pigs. J. Neurotrauma 13(3), 171-180.

Panjabi, M., Maak, T., Ivancic, P., and Ito, S. (2006). Dynamic intervertebral foramen narrowing during simulated rear impact. Spine 31(5), E128-134.

Pedowitz, R., Garfin, S., Massie, J., Hargens, A., Swenson, M., Myers, R., and Rydevik, B. (1992). Effects of magnitude and duration of compression on spinal nerve root conduction. Spine 17, 194-199.

Romero-Sandoval, E., Chai, N., Nutile-McMenemy, N., and DeLeo, J. (2008). A comparison of spinal Iba1 and GFAP expression in rodent models of acute and chronic pain. Brain Res. 1219, 116-126.

Rothman, S., Kreider, R., and Winkelstein, B. (2005). Spinal neuropeptide responses in persistent and transient pain following cervical nerve root injury. Spine 30, 2491-2496.

Rothman, S., and Winkelstein, B. (2007). Chemical and mechanical nerve root insults induce differential behavioral sensitivity and glial activation that are enhanced in combination. Brain Res. 1181, 30-43.

Sapunar, D., Modric-Jednacak, K., Grkovic, I., Michalkiewicz, M., and Hogan, Q. (2005). Effect of peripheral axotomy on painrelated behavior and dorsal root ganglion neurons excitability in NPY transgenic rats. Brain Res. 1063, 48-58.

Stuber, K. (2005). Cervical collars and braces in athletic brachial plexus injury and excessive cervical motion preven- tion: a review of the literature. J. Can. Chiropr. Assoc. 49(3), 216-222.

Swanik, C., Henry, T., and Lephart, S. (1996). Chronic brachial plexopathies and upper extremity proprioception and strength. J. Athl. Train. 31(2), 119-124.

Tominaga, Y., Maak, T., Ivancic, P., Panjabi, M., and Cunningham, B. (2006). Head-turned rear impact causing dynamic cervical intervertebral foramen narrowing: implications for ganglion and nerve root injury. J. Neurosurg. Spine 4(5), 380-387.

Wall, E., Kwan, M., Rydevik, B., Woo, S., and Garfin, S. (1991). Stress relaxation of a peripheral nerve. J. Hand Surg. 16, 859863.

Wall, P., and Melzack, R. (1994). Textbook of Pain, 3rd edition. Churchill Livingstone: London.

Winkelstein, B., and DeLeo, J. (2002). Nerve root injury severity differentially modulates spinal glial activation in a rat lumbar radiculopathy model: considerations for persistent pain. Brain Res. 956, 294-301.

Yoshizawa, H., Kobayashi, S., and Kubota, K. (1989). Effects of compression on intraradicular blood flow in dogs. Spine 15, $1220-1225$.

Zhuang, Z., Gerner, P., Woolf, C., and Ji, R. (2005). ERK is sequentially activated in neurons, microglia, and astrocytes by spinal nerve ligation and contributes to mechanical allodynia in this neuropathic pain model. Pain 114, 149-159.

Zimmermann, M. (1983). Ethical guidelines for investigations of experimental pain in conscious animals. Pain 16, 109-110.

Address correspondence to: Beth Winkelstein, Ph.D. Department of Bioengineering University of Pennsylvania 240 Skirkanich Hall 210 S. 33rd Street Philadelphia, PA 19104-6392

E-mail: winkelst@seas.upenn.edu 